\title{
PERIODIC BOEHMIANS II
}

\section{DENNIS NEMZER}

\begin{abstract}
A space of periodic generalised functions, called boehmians, is investigated. The space of boehmians contains all periodic distributions. It is known that not every hyperfunction is a boehmian. We show that the converse is also true. We present some theorems which give sufficient conditions for a sequence of complex numbers to be the Fourier coefficients of a boehmian. Sufficient conditions (in terms of the Fourier coefficients) are obtained for a sequence of boehmians to converge. As an application, a Dirichlet problem is discussed.
\end{abstract}

\section{INTRODUCTION}

Generalised functions on the unit circle have been successfully classified by the behaviour of their Fourier coefficients (see $[1,2,3,4,8]$ ). For example, a sequence of complex numbers $\left\{\zeta_{n}\right\}_{-\infty}^{\infty}$ is the Fourier coefficients of a Schwartz distribution if the $\zeta_{n}$ 's grow no faster than a polynomial in $n$. A sequence $\left\{\zeta_{n}\right\}_{-\infty}^{\infty}$ is the Fourier coefficients of a hyperfunction if $\varlimsup_{n}\left|\zeta_{n}\right|^{1 / n} \leqslant 1$. Also, every sequence of complex numbers is the Fourier coefficients of a Mikusinski operator. In this note we will consider a class of periodic generalised functions called Boehmians $([5,6,7])$. Classifying the space of Boehmians by the behaviour of their Fourier coefficients appears to be more complicated than with other spaces of generalised functions (for example, distributions, hyperfunctions, Mikusinski operators). The Fourier coefficients of a Boehmian must adhere to some growth restrictions (see Theorem B). However, as we shall show in Theorem 3.1, a subsequence of the Fourier coefficients may be unrestricted.

The following results about the Fourier coefficients of a Boehmian are known (see [6]).

THEOREM A. Let $\omega$ be a real-valued even function defined on the integers $Z$ such that $0=\omega(0) \leqslant \omega(n+m) \leqslant \omega(n)+\omega(m)$ for all $n, m \in Z$ and $\sum_{n=1}^{\infty} \omega(n) / n^{2}<$ $\infty$. Suppose $\left\{\zeta_{n}\right\}_{-\infty}^{\infty}$ is a sequence of complex numbers such that $\zeta_{n}=O\left(e^{\omega(n)}\right)$ as $|n| \rightarrow \infty$; then $\left\{\zeta_{n}\right\}_{-\infty}^{\infty}$ is the Fourier coefficients of a Boehmian.

TheOREM B. Let $\omega: Z \rightarrow R$ be an increasing function for $n=0,1,2, \ldots$ and $\sum_{n=1}^{\infty} \omega(n) / n^{2}=\infty$. Suppose $\left\{\zeta_{n}\right\}_{-\infty}^{\infty}$ is a sequence of complex numbers such that there

Received 22 October 1990

Copyright Clearance Centre, Inc. Serial-fee code: 0004-9729/91 \$A2.00+0.00. 
exist positive $A, M$, and $\varepsilon$ such that $\left|\zeta_{n}\right| \geqslant A e^{e \omega(n)}$ for all $n \geqslant M$. Then $\left\{\zeta_{n}\right\}_{-\infty}^{\infty}$ is not the Fourier coefficients of a Boehmian.

If we let $\omega(n)=n / \ln n$ for $n=2,3, \ldots$ and $\omega(n)=0$ otherwise then, by Theorem B, $\{\omega(n)\}_{-\infty}^{\infty}$ is not the Fourier coefficients of a Boehmain. But $\left\lceil|\omega(n)|^{1 / n}=1\right.$ and hence $\{\omega(n)\}_{-\infty}^{\infty}$ is the Fourier coefficients of a hyperfunction ([4]). Thus the space of Boehmians does not embrace the space of hyperfunctions. It can be shown that if the sequence $\left\{\zeta_{n}\right\}_{-\infty}^{\infty}$ is the Fourier coefficients of a Boehmian $x$ that satisfies the hypothesis of Theorem A (for some function $\omega$ ), then $\varlimsup_{\lim }\left|\zeta_{n}\right|^{1 / n} \leqslant 1$ and hence $x$ is a hyperfunction. Thus, the only known Boehmians are hyperfunctions.

In [6] the author poses the question "is the space of Boehmians contained in the space of hyperfunctions?" We shall give an example that will show the answer to this question is no.

In Section 2 we construct the space of Boehmians and state some known results ([6]). In Section 3 we present a theorem (Theorem 3.1) which improves upon Theorem $A$ and also gives rise to an example of a Boehmian which is not a hyperfunction. Then conditions are given on the Fourier coefficients of a sequence $\left\{x_{n}\right\}_{1}^{\infty}$ of Boehmians that ensure the convergence of the sequence (see Theorem 3.2). In Section 4 an application to a Dirichlet problem is discussed.

\section{Preliminaries}

$C(\Gamma)\left(L^{1}(\Gamma)\right)$ is the collection of all continuous (integrable) complex-valued functions on the unit circle $\Gamma$.

The convolution of $f$ and $g$ in $C(\Gamma)$ is denoted by juxtaposition. Thus,

$$
(f g)(t)=\frac{1}{2 \pi} \int_{-\pi}^{\pi} f(t-u) g(u) d u
$$

A sequence of continuous real-valued functions, $\left\{\delta_{n}\right\}_{1}^{\infty}$, is called a delta sequence if the following conditions are satisfied:

(i) For each $n, 1 / 2 \pi \int_{-\pi}^{\pi} \delta_{n}(t) d t=1$.

(ii) For each $n$ and all $t, \delta_{n}(t) \geqslant 0$.

(iii) Given a neighbourhood $V$ of 1 , there exists a positive integer $N$ such that for all $n \geqslant N$, the support of $\delta_{n}$ is contained in $V$.

The collection of delta sequences will be denoted by $\Delta$.

Definition 2.1: Let $A \subseteq C^{N}(\Gamma) \times \Delta\left(C^{N}(\Gamma)\right.$ is the set of sequences of elements of $C(\Gamma)$ ) be defined by

$$
A=\left\{\left(\left\{f_{n}\right\},\left\{\delta_{n}\right\}\right): \text { for each } k \text { and each } m, f_{k} \delta_{m}=f_{m} \delta_{k}\right\}
$$


Two elements $\left(\left\{f_{n}\right\},\left\{\delta_{n}\right\}\right)$ and $\left(\left\{g_{n}\right\},\left\{\sigma_{n}\right\}\right)$ of $A$ are said to be equivalent if for all $k$ and $m, f_{k} \sigma_{m}=g_{m} \delta_{k}$. A straightforward calculation shows that this is an equivalence relation on $A$. The equivalence classes are called periodic Boehmians.

Definition 2.2: The space of periodic Boehmians, denoted by $\beta$, is defined by $\beta=\left\{\left[\left\{f_{n}\right\} /\left\{\delta_{n}\right\}\right]:\left(\left\{f_{n}\right\},\left\{\delta_{n}\right\}\right) \in A\right\}$. For convenience a typical element of $\beta$ will be written as $x=f_{n} / \delta_{n}$.

The space of Schwartz distributions ([8]) can be viewed as a subspace of $\beta$ by identifying $u$ with $u^{*} \delta_{n} / \delta_{n}$, where $\left\{\delta_{n}\right\} \in C_{\infty}^{N} \cap \Delta$ and $u^{*} \delta_{n}$ denotes the convolution of $u$ and $\delta_{n}$ as distributions.

The Fourier coefficients of an $L^{1}(\Gamma)$ function are defined in the usual way. That is, if $f \in L^{1}(\Gamma)$, then

$$
C_{k}(f)=\frac{1}{2 \pi} \int_{-\pi}^{\pi} f(t) e^{-i k t} d t, \quad \text { for } k=0, \pm 1, \pm 2, \ldots .
$$

Definition 2.3: Let $x=f_{n} / \delta_{n} \in \beta$. For $k=0, \pm 1, \pm 2, \ldots$ define $C_{k}(x)=$ $C_{k}\left(f_{n}\right) / C_{k}\left(\delta_{n}\right)$, where for fixed $k, n$ is the smallest index such that $C_{k}\left(\delta_{n}\right) \neq 0$.

DEFINITION 2.4: A sequence $\left\{x_{n}\right\}_{1}^{\infty}$ of Boehmians is said to be $\Delta$-convergent to $x$, denoted by $\Delta-\lim x_{n}=x$, if there exists a delta sequence $\left\{\delta_{n}\right\}_{1}^{\infty}$ such that $\left(x_{n}-x\right) \delta_{n} \in C(\Gamma)$ for all $n$ and $\left(x_{n}-x\right) \delta_{n} \rightarrow 0$ uniformly as $n \rightarrow \infty$.

In [5] Mikusinski proved that $\beta$ endowed with $\Delta$-convergence is a complete topological vector space in which the topology is induced by an invariant metric.

The proofs of the next two theorems may be found in [6].

THEOREM 2.5. Let $x, x_{n} \in \beta$, for $n=1,2, \ldots$ If $\Delta-\lim x_{n}=x$, then for each $k, \lim _{n} C_{k}\left(x_{n}\right)=C_{k}(x)$.

THEOREM 2.6. For each $x \in \beta, x=\Delta-\lim _{n} \sum_{k=-n}^{n} C_{k}(x) e^{i k t}$.

\section{The MAIN RESUlT}

THEOREM 3.1. Let $\omega$ be a real-valued even function defined on the integers $Z$ such that $0=\omega(0) \leqslant \omega(n+m) \leqslant \omega(n)+\omega(m)$ for all $n, m \in Z$ and $\sum_{n=1}^{\infty} \omega(n) / n^{2}<\infty$. Suppose that the set of positive integers is partitioned into two disjoint sets $\left\{t_{n}\right\}_{1}^{\infty}$ and $\left\{s_{n}\right\}_{1}^{\infty}$ with $\sum_{n=1}^{\infty} 1 / t_{n}<\infty$. If $\left\{\zeta_{n}\right\}_{-\infty}^{\infty}$ is a sequence of complex numbers such that $\zeta_{ \pm s_{n}}=O\left(e^{\omega\left(o_{n}\right)}\right)$ as $n \rightarrow \infty$, then $\left\{\zeta_{n}\right\}_{-\infty}^{\infty}$ is the sequence of Fourier coefficients for some Boehmian.

Proof: For $n=1,2, \ldots$ let $\varphi_{n}(t)=t_{n} / 2 \pi$ for $|t| \leqslant \pi / t_{n}$ and zero otherwise. Let $\tilde{\varphi}_{n}$ for $n=1,2, \ldots$ be the $2 \pi$-periodic extension of $\varphi_{n}$. For $n=1,2, \ldots$ let 
$\delta_{n}=\prod_{j=n}^{\infty} \tilde{\varphi}_{j}$ (where the product is convolution). Since $\sum_{n=1}^{\infty} 1 / t_{n}<\infty$, it can be proven (see [5]) that $\left\{\delta_{n}\right\}_{1}^{\infty}$ is a delta sequence. Since, for each $k$ and all $n, C_{k}\left(\tilde{\varphi}_{n}\right)=$ $\alpha_{k, n} \sin \left(k \pi / t_{n}\right)$ (where $\alpha_{k, n}$ is a constant), we see that $C_{t_{k}}\left(\delta_{n}\right)=C_{-t_{k}}\left(\delta_{n}\right)=0$ for all $k \geqslant n$. Now, there exists a delta sequence $\left\{\sigma_{n}\right\}_{1}^{\infty}$ such that, for each $n$, $C_{k}\left(\sigma_{n}\right)=O\left(e^{-\omega^{*}(k)}\right)$ as $|k| \rightarrow \infty$, where $\omega^{*}(k)=\omega(k)+\sqrt{|k|}$ (see proof of Theorem 4.2 in [6]). Let $\left\{\psi_{n}\right\}_{1}^{\infty}$ be the delta sequence defined by $\psi_{n}=\delta_{n} \sigma_{n}$ for $n=1,2, \ldots$ Define $f_{n}(t)=\sum_{j=-n}^{n} \zeta_{j} e^{i j t}$ for $n=1,2, \ldots$ Then for each $k$ and all $n,\left(f_{n} \psi_{k}\right)(t)=$ $\sum_{j=-n}^{n} \zeta_{j} C_{j}\left(\psi_{k}\right) e^{i j t}$. Since, for each $k, \zeta_{j} C_{j}\left(\psi_{k}\right)=O\left(j^{-2}\right)$ as $|j| \rightarrow \infty$, for each $k$ the sequence of continuous functions $\left\{f_{n} \psi_{k}\right\}_{1}^{\infty}$ converges uniformly as $n \rightarrow \infty$. Hence, $\Delta-\lim f_{n}=\Delta-\lim _{n}\left(f_{n} \psi_{k} / \psi_{k}\right)=x \in \beta$ (see [5]). By Theorem 2.5, for each $m$, $C_{m}(x)=\lim _{n} C_{m}\left(f_{n}\right)=\zeta_{m}$ and hence the theorem follows.

REMARKS. (i) The above theorem shows that $\beta$ is not contained in the set of hyperfunctions. Indeed, if $\left\{\zeta_{n}\right\}_{-\infty}^{\infty}$ is chosen appropriately (that is, $\overline{\lim }\left|\zeta_{n}\right|^{1 / n}>1$ ) and $x$ is the Boehmian having Fourier coefficients $\left\{\zeta_{n}\right\}_{-\infty}^{\infty}$, then $x$ is not a hyperfunction ([4]).

(ii) Theorem 3.1 may also be used to construct a Boehmian that is not a Beurling distribution ([2]).

The next theorem gives a partial converse to Theorem 2.5.

Theorem 3.2. Suppose $\left\{x_{n}\right\}_{1}^{\infty}$ is a sequence of Boehmians such that

(i) there exist a constant $M$ and a Boehmian $y$ such that $\left|C_{k}\left(x_{n}\right)\right| \leqslant$ $M\left|C_{k}(y)\right|$ for all $n$ and all $k$;

(ii) for each $k C_{k}\left(x_{n}\right) \rightarrow \zeta_{k}$ as $n \rightarrow \infty$.

Then $\left\{\zeta_{k}\right\}_{-\infty}^{\infty}$ is the Fourier coefficients of a Boehmian $x$. Moreover, $\Delta-\lim x_{n}=x$.

Proof: By conditions (i) and (ii) we have that

$$
\left|\zeta_{k}\right| \leqslant M\left|C_{k}(y)\right| \text { for all } k \text {. }
$$

We may assume that for some delta sequence $\left\{\delta_{n}\right\}_{1}^{\infty}, y \delta_{n} \in C^{\infty}$ for all $n$. For if $\left\{\sigma_{n}\right\}_{1}^{\infty}$ is a delta sequence such that $y \sigma_{n} \in C(\Gamma)$ for all $n$, let $\left\{\psi_{n}\right\}_{1}^{\infty}$ be an infinitely differentiable delta sequence and take $\delta_{n}=\sigma_{n} \psi_{n}$ for $n=1,2, \ldots$ Thus

$$
\text { for each } n, C_{k}\left(y \delta_{n}\right)=O\left(k^{-2}\right) \text { as }|k| \rightarrow \infty \text {. }
$$

Let $f_{n}(t)=\sum_{j=-n}^{n} \zeta_{j} e^{i j t}$ for $n=0,1,2, \ldots$ Then for all $n$ and $m,\left(f_{n} \delta_{m}\right)(t)=$ $\sum_{j=-n}^{n} \zeta_{j} C_{j}\left(\delta_{m}\right) e^{i j t}$ and from (3.1) and (3.2) we see that for each $m, \alpha_{j} C_{j}\left(\delta_{m}\right)=O\left(j^{-2}\right)$ 
as $|j| \rightarrow \infty$. Thus, for each $m$ the sequence of continuous functions $\left\{f_{n} \delta_{m}\right\}_{0}^{\infty}$ converges uniformly as $n \rightarrow \infty$. Hence, $\Delta-\lim f_{n}=\Delta-\lim _{n}\left(f_{n} \delta_{m} / \delta_{m}\right)=x \in \beta$ (see [5]). It now follows from Theorem 2.5 that for each $k, C_{k}(x)=\lim _{n} C_{k}\left(f_{n}\right)=\zeta_{k}$.

To complete the proof of the theorem it suffices to show that if

$$
\begin{gathered}
\lim _{n} C_{k}\left(x_{n}\right)=0 \text { for all } k, \text { and } \\
\left|C_{k}\left(x_{n}\right)\right| \leqslant M\left|C_{k}(y)\right| \text { for all } n \text { and } k,
\end{gathered}
$$

then $\Delta-\lim x_{n}=0$.

Now, there exists a delta sequence $\left\{\delta_{n}\right\}_{1}^{\infty}$ such that $y \delta_{m} \in C^{\infty}$ for all $m$ and hence

$$
\text { for each } m, C_{j}\left(y \delta_{m}\right)=O\left(j^{-2}\right) \text { as }|j| \rightarrow \infty \text {. }
$$

Thus, by (3.4) and (3.5), for each $m$ and all $n, C_{j}\left(x_{n} \delta_{m}\right)=O\left(j^{-2}\right)$ as $|j| \rightarrow \infty$. Thus, for each $n$ and all $m\left\{C_{j}\left(x_{n} \delta_{m}\right)\right\}_{-\infty}^{\infty}$ is the Fourier coefficients of a continuous function. Moreover, for each $m$, and all $n\left(x_{n} \delta_{m}\right)(t)=\sum_{-\infty}^{\infty} C_{j}\left(x_{n} \delta_{m}\right) e^{i j t}$.

Now, fix $m$. Given an $\varepsilon>0$, it follows from (3.5) that there exists an $N$ such that

$$
\sum_{|j|>N} M\left|C_{j}\left(y \delta_{m}\right)\right|<\varepsilon / 2
$$

By (3.3), there exists a $T$ such that

$$
\text { for each } n>T,\left|C_{j}\left(\delta_{m}\right)\right|\left|C_{j}\left(x_{n}\right)\right|<\varepsilon /(4 N+2) \text { for } j=0, \pm 1, \pm 2, \ldots, \pm N
$$

It follows from (3.6) and (3.7) that for each $n>T,\left|\left(x_{n} \delta_{m}\right)(t)\right|<\varepsilon$ for all $t$. That is, for each $m, x_{n} \delta_{m} \rightarrow 0$ uniformly as $n \rightarrow \infty$. Hence $\Delta-\lim x_{n}=0$ (see [5]) and the theorem is established.

Corollary 3.3. If $\left\{\zeta_{n}\right\}_{-\infty}^{\infty}$ is a sequence of complex numbers such that $\zeta_{n}=$ $O\left(C_{n}(x)\right)$ as $|n| \rightarrow \infty$ for some $x \in \beta$, then $\left\{\zeta_{n}\right\}_{-\infty}^{\infty}$ is the Fourier coefficients of a Boehmian $y$. Moreover, $y=\Delta-\lim _{n} \sum_{j=-n}^{n} \zeta_{j} e^{i j t}$.

Proof: The proof follows immediately by applying Theorem 3.2 to the sequence $\left\{f_{n}\right\}_{0}^{\infty}$ of Boehmians, where $f_{n}(t)=\sum_{j=-n}^{n} \zeta_{j} e^{i j t}$ for $n=0,1,2, \ldots$ 


\section{A DirichleT PROBLEM FOR THE DISK}

The Dirichlet problem (in polar coordinates) for the disk is to find a function $u(r, \theta)$ such that

$$
\begin{aligned}
r^{2} u_{r r}+r u_{r}+u_{\theta \theta}=0, & 0<r<1, \quad-\infty<\theta<\infty \\
\text { and } u(1, \theta) & \stackrel{\circ}{=} g(\theta), \quad-\infty<\theta<\infty \quad \text { (where } g \text { is a given periodic function). }
\end{aligned}
$$

The solution $u$ of the above Dirichlet problem may be interpreted physically as the steady-state temperature at the point $(r, \theta)$ in the disk when the boundary temperature is given by $g(\theta)$.

In order to formulate a more general Dirichlet problem we need some preliminaries.

Definition 4.1: Let $I=(a, b)$ be an interval. A Boehmian-valued function $F: I \rightarrow \beta$ is said to have a derivative equal to $F^{\prime}(\lambda)(\lambda \in I)$ if for any $\lambda_{n} \in I$ such that $\lambda_{n} \neq \lambda$ for $n=1,2, \ldots$ and $\lambda_{n} \rightarrow \lambda$ as $n \rightarrow \infty$ we have $\Delta-\lim \left(F\left(\lambda_{n}\right)-F(\lambda)\right) /\left(\lambda_{n}-\lambda\right)=F^{\prime}(\lambda)$. In general, $F^{(n)}(\lambda)=\left(F^{(n-1)}(\lambda)\right)^{\prime}$ for $n=2,3, \ldots$

The proof of the next theorem follows directly from Theorem 2.5.

TheOREM 4.2. For each $k$ and all $n, C_{k}\left(F^{(n)}(\lambda)\right)=d^{n} / d \lambda^{n}\left(C_{k} \circ F\right)(\lambda)$ for all $\lambda$.

The $n$th order differentiation Boehmian is given by $s^{n}=\delta_{j}^{(n)} / \delta_{j}$ for $n=1,2, \ldots$ (where $\left\{\delta_{j}\right\}_{1}^{\infty} \in C_{\infty}^{N} \cap \Delta$ ). A straightforward exercise shows that if $f$ is an $n$-times continuously differentiable function then $s^{n} f=f^{(n)}$.

We now consider the following Dirichlet problem.

Given an $x \in \beta$, find a Boehmian-valued function $F:(0,1) \rightarrow \beta$ such that

$$
r^{2} F^{\prime \prime}+r F^{\prime}+s^{2} F=0 \text { for } 0<r<1 \text { and } \Delta-\lim _{r \rightarrow 1^{-}} F(r)=x
$$

Theorem 4.3. (Existence). For each $x \in \beta$ there exists a solution to (4.2).

PROOF: Let $F(r)=\Delta-\lim _{n} \sum_{j=-n}^{n} C_{j}(x) r^{|j|} e^{i j t}=\sum_{-\infty}^{\infty} C_{j}(x) r^{|j|} e^{i j t}, 0<r<1$. By Corollary 3.3, the above limit exists and $F(r) \in \beta$ for all $r$.

Let $0<r<1$ and $\left\{r_{n}\right\}_{1}^{\infty}$ be a sequence in $(0,1)$ such that $r_{n} \rightarrow r$ as $n \rightarrow \infty$ and $r_{n} \neq r$ for all $n$. Now

$$
\frac{F\left(r_{n}\right)-F(r)}{r_{n}-r}=\sum_{|j| \geqslant 1} C_{j}(x) \frac{r_{n}^{|j|}-r^{|j|}}{r_{n}-r} e^{i j t}=\sum_{|j| \geqslant 1} C_{j}(x)\left(\sum_{k=0}^{|j|-1} r_{n}^{|j|-k-1} r^{k}\right) e^{i j t}
$$


For each $m \neq 0$ and all $n$

$$
\left|C_{m}\left(\frac{F\left(r_{n}\right)-F(r)}{r_{n}-r}\right)\right|=\left|C_{m}(x) \sum_{k=0}^{|m|-1} r_{n}^{|m|-k-1} r^{k}\right| \leqslant|m|\left|C_{m}(x)\right|=\left|C_{m}(s x)\right| .
$$

Also, for each $m \neq 0$

$$
C_{m}\left(\frac{F\left(r_{n}\right)-F(r)}{r_{n}-r}\right)=C_{m}(x) \sum_{k=0}^{|m|-1} r_{n}^{|m|-k-1} r^{k} \rightarrow C_{m}(x)|m| r^{|m|-1} \text { as } n \rightarrow \infty .
$$

So, by Theorem 3.2 and Corollary 3.3, $\Delta-\lim \left(F\left(r_{n}\right)-F(r)\right) /\left(r_{n}-r\right)=$ $\sum_{|j| \geqslant 1} C_{j}(x)|j| r^{|j|-1} e^{i j t}$. Hence,

$$
F^{\prime}(r)=\sum_{|j| \geqslant 1} C_{j}(x)|j| r^{|j|-1} e^{i j t}, \quad 0<r<1
$$

Similarly,

$$
F^{\prime \prime}(r)=\sum_{|j| \geqslant 2} C_{j}(x)|j|(|j|-1) r^{|j|-2} e^{i j t}, \quad 0<r<1
$$

By using (4.3), (4.4), and Theorem 2.6 we obtain $r^{2} F^{\prime \prime}+r F^{\prime}+s^{2} F=0$ for $0<r<1$.

In order to complete the proof we need to show that $\Delta-\lim _{r \rightarrow 1^{-}} F(r)=x$. For each $k$ and all $0<r<1,\left|C_{k}(F(r))\right|=\left|C_{k}(x) r^{|k|}\right| \leqslant\left|C_{k}(x)\right|$. Also, for all $k, C_{k}(F(r))=$ $C_{k}(x) r^{|k|} \rightarrow C_{k}(x)$ as $r \rightarrow 1^{-}$. Therefore, by applying Theorem 3.2, the proof is complete.

REMARK. If $x \in C(\Gamma) \subseteq \beta$, then (as seen in the proof of Theorem 4.3) the solution $u(r, \theta)=\sum_{-\infty}^{\infty} C_{k}(x) r|k| e^{i k \theta}$ of (4.2) is the classical solution of the Dirichlet problem (4.1).

A function $F:(a, b) \rightarrow \beta$ will be called weakly bounded if for each $k$ the set $\left\{\left(C_{k} \circ F\right)(\lambda): \lambda \in(a, b)\right\}$ is bounded.

Theorem 4.4. (Uniqueness). For each $x \in \beta$ there exists at most one weakly bounded solution to (4.2).

Proof: Suppose that $r^{2} F^{\prime \prime}+r F^{\prime}+s^{2} F=0,0<r<1$, and $\Delta-\lim _{r \rightarrow 1^{-}} F(r)=0$. By applying Theorem 4.2 to the above we obtain for $k= \pm 1, \pm 2, \ldots,\left(C_{k} \circ F\right)(r)=$ $A_{k} r^{k}+B_{k} r^{-k}$ and $\left(C_{0} \circ F\right)(r)=A_{0}+B_{0} \ln r, 0<r<1$, (where $A_{k}$ and $B_{k}$ are constants). Since $F$ is weakly bounded $\left(C_{k} \circ F\right)(r)=A_{k} r^{k}$ for $k=0, \pm 1, \pm 2, \ldots$ 
Therefore, by applying Theorem 2.5 and the condition $\Delta-\lim _{r \rightarrow 1^{-}} F(r)=0$, we obtain that $A_{k}=0$ for $k=0, \pm 1, \pm 2, \ldots$ Hence, $F(r)=0$ for $0<r<1$.

Let $H=\left\{x \in \beta: \varlimsup \lim \left|C_{k}(x)\right|^{1 / k} \leqslant 1\right\}$. Since $H$ can be identified with a subset of the space of hyperfunctions ([4]), elements of $H$ are called hyperboehmians.

The next regularity theorem is easily proved and hence its proof is omitted.

THEOREM 4.5. For each $x \in H$ there is a unique weakly bounded solution $F$ to (4.2). Moreover, if $u(r, \theta)=F(r), 0<r<1,-\infty<\theta<\infty$, then

(i) $u$ is an infinitely differentiable function.

(ii) $r^{2} u_{r r}+r u_{r}+u_{\theta \theta}=0,0<r<1$ and $-\infty<\theta<\infty$, and $\Delta-\lim _{r \rightarrow 1^{-}} u(r, \theta)=x$.

REMARK. It is not difficult to show that $u(r, \theta)=\sum_{-\infty}^{\infty} C_{k}(x) r^{|k|} e^{i k \theta}(x \in \beta)$ represents a harmonic function for $0<r<1$ and $-\infty<\theta<\infty$ if and only if $x$ is a hyperboehmian. Indeed, if $x \in \beta \backslash H$ then the solution $\sum_{-\infty}^{\infty} C_{k}(x) r^{|k|} e^{i k \theta}$ to (4.2) is not even a function of $\boldsymbol{\theta}$.

\section{References}

[1] T.K. Boehme and G. Wygant, 'Generalized functions on the unit circle', Amer. Math. Monthly 82 (1975), 256-261.

[2] M.G. Collier and J.A. Kelingos, 'Periodic Beurling distributions', Acta. Math. Hungar. 42 (1983), 261-278.

[3] V.I. Gorbacuk and M.L. Gorbacuk, 'Trigonometric series and generalized periodic functions', Soviet Math. Dokl. 23 (1981), 342-346.

[4] G. Johnson, 'Harmonic functions on the unit disk', Ilinois J. Math. 12 (1968), 366-385.

[5] P. Mikusinski, 'Convergence of Boehmians', Japan J. Math. 9 (1983), 159-179.

[6] D. Nemzer, 'Periodic Boehmians', Internat. J. Math. Math. Sci. 12 (1989), 685-692.

[7] D. Nemzer, 'Periodic generalized functions', Rocky Mountain J. Math. 20 (1990).

[8] L. Schwartz, Theorie des distributions (Herman, Paris, 1966).

Department of Mathematics

California State University, Stanislaus

Turlock California 95380

United States of America 